\title{
Etude des mécanismes du rinçage par un film tombant d'une surface verticale souillée par des solutions modèles et des produits laitiers - Modélisation du phénomène
}

\author{
J.P. TISSIER, M. LALANDE, G. CORRIEU
}

INRA, Laboratoire de Génie industriel alimentaire, B.P. 39, 59651 Villeneuve-d'Ascq Cedex, France

\section{Résumé}

Un dispositif expérimental simulant la paroi d'un tank de stockage est constitué d'une plaque verticale qui est souillée sur une hauteur de $2,25 \mathrm{~m}$ par $10 \mathrm{dm}^{3}$ de solution. La plaque est rincée par un film tombant homogène.

Les résultats suivants sont obtenus :

- La quantité de souillure restant sur la plaque après égouttage dépend de la viscosité de la solution de souillure.

- La cinétique du rinçage qui dépend de l'aptitude au rinçage des composants de la souillure est simulée par un modèle qui comprend trois phases. La première phase, d'ordre zéro, assure l'élimination des fortes épaisseurs de souillure. La deuxième phase, d'ordre un, assure l'élimination de souillures piégées dans les rugosités de la surface. La dernière phase, d'ordre supérieur à un, paraît contrôlée par des phénomènes de surface.

- Les petites molécules sont éliminées plus rapidement que les protéines et la matière grasse du lait. La vitesse d'élimination par rinçage crôt selon le composé dans l'ordre suivant : matière grasse de lait homogénéisé et micelles de caséine, matière grasse du lait non homogénéisé, protéines du lactosérum et caséine soluble, $\mathrm{NaOH}$ et $\mathrm{HNO}_{3}$, fluorescéinate de sodium, saccharose et lactose, $\mathrm{NaCl}$.

Un parallèle est établi entre le rinçage des tanks de stockage et celui des canalisations.

Mots clés : Rinçage - Film tombant - Mécanismes - Cinétique - Produits laitiers - Solution de nettoyage.

\section{Summary}

Study of rinsing mechanisms using a falling film

on a vertical surface soiled by model solutions

and dairy products - Process modeling.

An experimental device which comprised a $3 \mathrm{~m}$ high vertical plate was built to simulate the wall of a storage tank. The plate was soiled with a falling film of model soiling solution and rinsed by means of a homogenous falling film of osmosed water. A sampling device comprising a high speed sampler was used to quantify total soil removed by rinsing and rinsing kinetics. 
The main results obtained were :

- Soil amount remaining on the plate after dripping off depended on soiling solution viscosity in agreement with VAN Rossum's theory (1958). The composition of the soil removed by rinsing was similar to the composition of the soiling solution.

- Rinsing kinetics could be simulated by a model involving three phases. This model took into account plate rugosity, residual soiling and rinsing ability of deposit components. The first phase whose order was equal to zero controlled removal of soiling film overlaying plate rugosity. The second phase whose order was equal to one controlled removal of soiling film contained into plate rugosity. The third phase whose order was superior to one seemed to be controlled by surface phenomena.

- Small molecules were removed more rapidly than milk protein and fat. Rinsing removal rate increased according to compounds in the following order : homogenized milk fat and casein micelles, non homogenized milk fat, whey proteins and soluble casein, $\mathrm{NaOH}$ and $\mathrm{HNO}_{3}$, Na-fluorescein, sucrose and lactose, NaCl.

- A parallel was established between rinsing of storage tanks and rinsing of pipes.

Key words : Rinsing - Mechanism - Falling film - Kinetics - Dairy products - Cleaning solutions.

\section{Introduction}

Pour des raisons hygiéniques, les tanks de stockage utilisés dans l'industrie alimentaire doivent, après leur vidange, être nettoyés. Cette opération qui inclut généralement plusieurs rinçages, s'effectue par projection d'eau qui ruisselle sous forme d'un film tombant sur la surface souillée. Dans la pratique industrielle, le nettoyage et le rinçage des tanks sont le plus souvent effectués de façon empirique. L'optimisation de ces opérations nécessiterait de connaître les mécanismes et cinétiques qui les contrôlent.

L'étude du nettoyage de tanks de stockage ayant contenu du lait entier non homogénéisé (TISSIER et al., 1983a et b) a déjà permis de mettre en évidence quelques résultats intéressants :

- La quantité de souillure restant après vidange est très faible. Ainsi après 35 minutes d'égouttage il ne reste plus qu'une vingtaine de centimètres cubes de lait par mètre carré de surface souillée de tank. Un simple rinçage à l'eau permet d'éliminer environ $90 \%$ de la souillure, et même la totalité du lactose.

- Les différents composants de la souillure ne sont pas éliminés à la même vitesse pendant le rinçage : le lactose est éliminé plus rapidement que les protéines alors que la matière grasse présente un comportement intermédiaire.

- Le volume d'eau utilisé par unité de surface souillée détermine la concentration des différents composants de la souillure dans l'effluent, indépendamment du débit de rinçage.

- La nature de la solution de rinçage n'influence pas la rapidité du rinçage.

- Une boule de nettoyage efficace doit établir un film de solution de rinçage correct sur la totalité de la surface souillée.

- Le pré-rinçage peut être effectué par la seule action de ruissellement d'un film tombant. Néanmoins, la disparition des composants les plus difficiles à élimi- 
ner est accélérée par l'existence de jets qui, arrivant à différents niveaux de la surface, contribuent à augmenter la turbulence du film tombant.

Ces premiers résultats nous ont incités à étudier le comportement, pendant le rinçage, de différents produits laitiers et de solutions modèles afin de préciser les mécanismes qui contrôlent cette opération. Une telle démarche a déjà été effectuée, dáns le cas des canalisations et des appareils pleins et fermés tels que les échangeurs à plaques, par l'équipe de LONCIN (THOR et LONCIN, 1978 ; PLETT et Loncin, 1981 ; Plett, 1984) et par Nassauer et Kessler (1982). Avec ce type d'appareil, la composition de la souillure utilisée influe sur la cinétique de rinçage. Les principales études relatives à ce phénomène peuvent être résumées ainsi :

- La cinétique du rinçage est décomposée en plusieurs phases qui s'enchaînent. Le comportement des différents composants de la souillure diffère pendant la troisième phase qui est décrite par une cinétique du premier ordre dont la constante de vitesse dépend de la nature de chaque composant (THOR et Loncin, 1978). PletT (1984) met en évidence une quatrième phase qui semble contrôlée par un mécanisme de désorption de la paroi et dont la constante de vitesse est encore plus faible.

- La nature ionique des agents tensio-actifs utilisés ne permet pas de classer leur aptitude au rinçage car NASSAUER et Kessler (1982) et PLetT (1984) observent des comportements différents lors du rinçage de canalisations en acier inoxydable. Ainsi, des détergents non ioniques et des détergents amphotères apparaissent soit très difficiles, soit faciles à rincer alors que les deux détergents cationiques étudiés sont difficiles à rincer.

- NAssauer et Kessler (1982) montrent qu'une augmentation de la concentration de la solution de souillure initialement contenue dans une canalisation entraîne une augmentation de la concentration relative en souillure de l'effluent à la fin du rinçage.

Nous avons cherché à vérifier si ces phénomènes, mis en évidence sur des canalisations, sont également observables dans le cas des tanks de stockage. Pour effectuer cette étude, nous avons conçu un dispositif permettant de simuler la paroi d'un tank et nous avons utilisé différentes solutions de souillure (des produits modèles simples utilisés seuls ou en mélange à différentes concentrations, et des produits laitiers caractéristiques).

\section{Matériels et méthodes}

\section{A. Description du dispositif expérimental}

Le dispositif expérimental qui simule la paroi intérieure d'un tank de stockage est détaillé précédemment (TISSIER, 1984). Schématisé à la figure 1, ce dispositif est constitué d'une plaque verticale (a) de grande dimension ( $3 \mathrm{~m}$ de haut et 0,75 m de large), en acier inoxydable Z2CN18-10 (304 L) laminé à froid, brut de laminage « glacé » (2B). Une mesure de rugosité par coupe optique montre que la distance entre les crêtes et les creux est en moyenne de 3,5 micromètres (DAUFIN, communication personnelle). Deux cornières en acier inoxydable (g) situées au bord de la surface expérimentale canalisent le film tombant sur une largeur de 


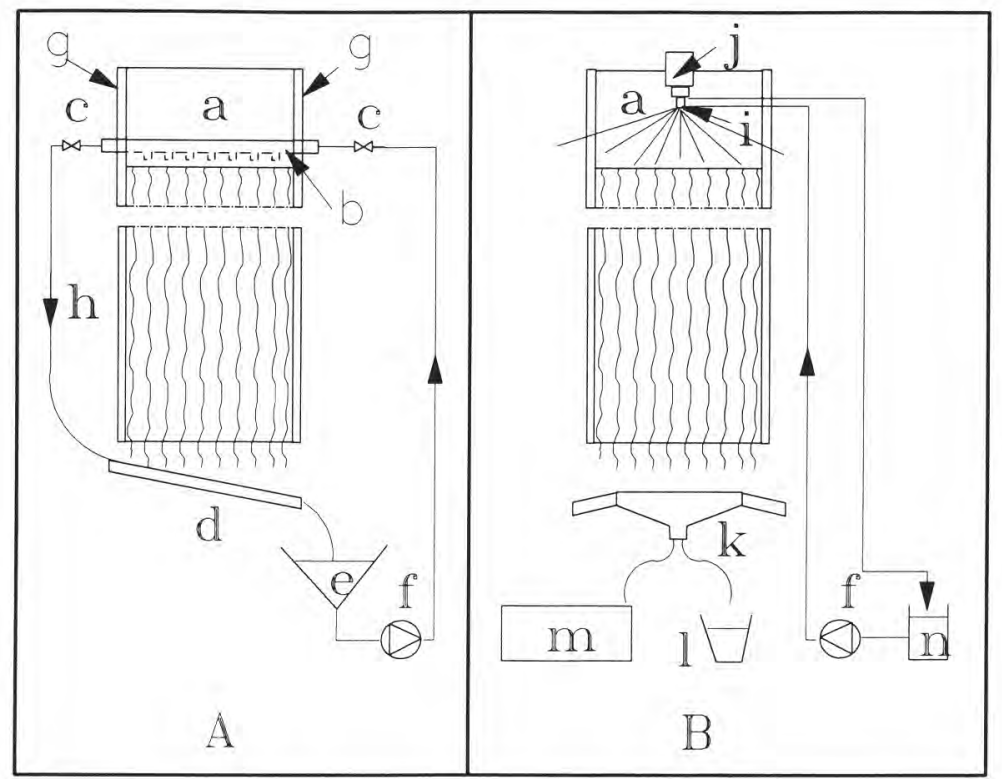

Fig. 1

Représentation schématique du dispositif utilisé

- pour souiller la plaque : $A$

- pour rincer la plaque : $B$

a. Plaque expérimentale

b. Dispositif de distribution de la souillure sur la plaque.

c. Robinets de réglage.

d. Gouttière permettant le recyclage de la souillure.

e. Réservoir contenant la souillure.

f. Pompe.

g. Cornière.

h. Canalisation de purge du distributeur.

$i$. Buse de distribution du film tombant de rinçage.

$j$. Vanne à trois voies.

$k$. Entonnoir de collecte de l'effluent.

l. Réservoir de collecte de l'échantillon moyen.

m. Collecteur d'échantillons.

$n$. Réservoir de stockage de la solution de rincage.

Schematic presentation of the experimental device.

- to soil the plate: $A$

- to rinse the plate: $B$

a. Experimental plate.

$b$. Distribution device to spread the soil on the plate.

c. Valves.

d. Gutter to recycle the soil.

e. Soil feed tank.

f. Pump.

g. Plate supports.

h. Draining of distribution device.

$i$. Spray of rinsing.

j. Three way valve.

$k$. Collector of rinsing solution.

l. Sampler for mean concentration.

$m$. Sampler for concentration/time curve.

$n$. Rinse water feed tank. 
$0,7 \mathrm{~m}$. Ainsi, les conditions hydrodynamiques de l'écoulement sont constantes sur la totalité de la hauteur de plaque. La plaque est souillée à l'aide du dispositif schématisé à la figure $1 \mathrm{~A}$ par la solution de souillure contenue dans un réservoir (e). Une pompe (f) alimente le distributeur (b) qui forme des jets. Ceux-ci étalés par un déflecteur forment un film tombant régulier de souillure qui ruisselle sur la plaque, sur une hauteur de $2,25 \mathrm{~m}$. Cette solution est recyclée par l'intermédiaire d'une gouttière (d). Ce dispositif expérimental a l'avantage d'être souillé par seulement $10 \mathrm{dm}^{3}$ de solution, alors qu'un tank nécessite un volume égal à sa contenance.

La plaque est rinçée au moyen du dispositif schématisé à la figure $1 \mathrm{~B}$. Une buse (i) projette un film plat d'eau osmosée d'épaisseur constante qui atteint la plaque verticale (a) environ $0,3 \mathrm{~m}$ au-dessus de la surface souillée et ruisselle en un film tombant homogène. La buse est portée par une vanne à 3 voies (j) qui commande le rinçage de la plaque et le recyclage de l'eau de rinçage dans le réservoir de stockage (n) de $80 \mathrm{dm}^{3}$.

Dans nos conditions expérimentales, l'épaisseur moyenne du film tombant de rinçage est de $0,50 \mathrm{~mm}$ et son débit exprimé par unité de largeur de plaque ( $\Gamma$ ) est de $3,7 \cdot 10^{-4} \mathrm{~m}^{2} \cdot \mathrm{s}^{-1}$. Le nombre de Reynolds (Re), calculé selon la relation 1 en fonction de $\Gamma$ et de la viscosité cinématique de l'eau de rinçage $(\gamma)$ est voisin de 1500 .

$$
\operatorname{Re}=4 \Gamma \cdot \gamma^{-1}
$$

Le régime d'écoulement peut donc être considéré comme au moins transitoire, plus vraisemblablement turbulent ( $\operatorname{Re}>1000$ à 2000 d'après BIRD et al., 1960).

Le dispositif utilisé pour recueillir l'effluent de rinçage comprend un entonnoir en acier inoxydable (k) qui collecte l'effluent sur une largeur de $0,5 \mathrm{~m}$ et élimine les $0,1 \mathrm{~m}$ de film tombant qui, à chaque extrémité, sont susceptibles d'être perturbés par l'effet de bord et la présence des cornières. A la sortie de l'entonnoir de recueil, un diviseur répartit le film en deux fractions identiques. Une des fractions fournit un échantillon représentatif de l'ensemble du rinçage. L'autre fraction, recueillie par le collecteur d'échantillons décrit préalablement (TISSIER et al., 1983a) permet de suivre l'évolution de la concentration de l'effluent de rinçage en fonction de la durée du processus et de déterminer sa cinétique. Le calcul montre que la cinétique du rinçage n'est que très faiblement influencée par la dispersion des temps de séjour du système de prélèvement qui correspond en première approximation à celle d'un système composé d'un réacteur piston de $150 \mathrm{~cm}^{3}$ placé en série avec un mélangeur parfait de $30 \mathrm{~cm}^{3}$ (TISsIER, 1984).

\section{B. Protocole expérimental d'un essai}

La plaque, préalablement nettoyée par une solution de soude à $20 \mathrm{~g} .1^{-1}$ puis rincée par de l'eau osmosée, est souillée pendant 5 minutes. Pendant les 35 minutes d'égouttement qui suivent, le dispositif de distribution de la souillure est remplacé par celui de rinçage et de recueil de l'effluent. Le rinçage commence lorsque la vanne 《 $\mathrm{j}$ » est commutée ; le démarrage du collecteur d'échantillons est simultané. Le poids total d'effluent collecté permet de calculer le débit réel de la solution de rinçage sur la surface expérimentale. Le poids de chacun des échantillons recueillis par le collecteur est mesuré afin de normaliser l'origine des temps de 


\section{TABLEAU 1}

Caractérisation des différentes souillures. Comparaison entre la quantité de souillure restant sur la plaque après égouttage, calculée selon la théorie de VAN Rossum (1958), et la quantité de souillure éliminée par rinçage.

Composition and characteristics of the different soils. Comparison between the theoretical soil amounts remaining on the plate after dropping, calculated by VAN RossUm's theory (1958), and the soil amounts removed by rinsing.

\begin{tabular}{|c|c|c|c|c|c|}
\hline \multicolumn{3}{|c|}{$\begin{array}{l}\text { Caractérisation des solutions } \\
\text { de souillure }\end{array}$} & \multicolumn{3}{|c|}{$\begin{array}{l}\text { Quantité moyenne } \\
\text { de souillure de } \\
\text { la plaque }\left(\mathrm{cm}^{3} \cdot \mathrm{m}^{-2}\right)\end{array}$} \\
\hline \multirow{2}{*}{ Composition } & \multirow{2}{*}{$\begin{array}{c}\mathrm{C}_{\mathrm{o}} \\
\mathrm{g} .1^{-1}\end{array}$} & \multirow{2}{*}{$\begin{array}{c}\gamma .10^{6} \\
\mathrm{~m}^{2} \cdot \mathrm{s}^{-1}\end{array}$} & \multirow{2}{*}{$\begin{array}{l}\text { Théo- } \\
\text { rique * }\end{array}$} & \multicolumn{2}{|c|}{ Expérimental } \\
\hline & & & & M & E.T.M. \\
\hline \multicolumn{6}{|c|}{ Composés simples et homogènes } \\
\hline 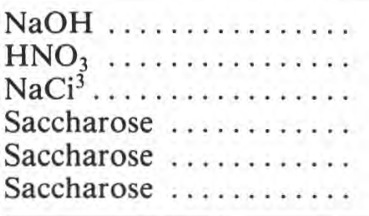 & $\begin{array}{r}20 \\
14 \\
20 \\
50 \\
150 \\
480\end{array}$ & $\begin{array}{c}1,09 \\
1,00 \\
1,02 \\
1,14 \\
1,6 \\
12,7\end{array}$ & $\begin{array}{r}7,3 \\
7,0 \\
7,1 \\
7,5 \\
8,8 \\
24,9\end{array}$ & $\begin{array}{r}10,9 \\
5,2 \\
2,6 \\
3,2 \\
3,7 \\
25,9\end{array}$ & $\begin{array}{l}1,8 \\
0,3 \\
0,9 \\
1,1 \\
1,5 \\
1,1\end{array}$ \\
\hline $\begin{array}{c}\text { Mélange } 1 \stackrel{\mathrm{NaCl}}{\text { Fluorescéine }} \\
\text { Moyenne }\end{array}$ & $\begin{array}{l}100 \\
0,2\end{array}$ & 1,2 & 7,4 & $\begin{array}{l}9,7 \\
9,4 \\
9,6\end{array}$ & $\begin{array}{l}2,8 \\
1,2 \\
1,9\end{array}$ \\
\hline $\begin{array}{c}\text { Saccharose } \\
\text { Mélange } 2 \\
\begin{array}{c}\text { Fluorescéine } \\
\text { Moyenne }\end{array} \\
\text { NaCl }\end{array}$ & $\begin{array}{l}480 \\
100 \\
0,2\end{array}$ & 14,0 & 26,2 & $\begin{array}{l}44,5 \\
43,7 \\
37,2 \\
41\end{array}$ & $\begin{array}{l}4,6 \\
3,7 \\
3,0 \\
4,8\end{array}$ \\
\hline \multicolumn{6}{|c|}{ Produits laitiers } \\
\hline $\begin{array}{l}\text { Lait cru Matière grasse } \\
\text { entier non } \\
\text { homogénéisé } \quad \text { Protéines } \\
\text { Moctose } \\
\text { Moyenne }{ }^{*}\end{array}$ & $\begin{array}{l}40 \\
35 \\
50\end{array}$ & 3,6 & 13,3 & $\begin{array}{l}13,3 \\
15,3 \\
17,5 \\
15,4\end{array}$ & $\begin{array}{l}0,7 \\
1,8 \\
0,7 \\
2,1\end{array}$ \\
\hline $\begin{array}{l}\text { Lait UHT Matière grasse } \\
\text { entier } \quad \text { Protéines } \\
\text { homogénéisé } \quad \text { Lactose } \\
\text { Moyenne * } \\
\end{array}$ & $\begin{array}{l}35 \\
35 \\
50\end{array}$ & 3,6 & 13,3 & $\begin{array}{l}14,5 \\
15,0 \\
14,9 \\
14,8\end{array}$ & $\begin{array}{l}0,4 \\
0,7 \\
2,7 \\
1,4\end{array}$ \\
\hline $\begin{array}{lr}\begin{array}{l}\text { Caséine } \\
\text { soluble }+ \\
\text { Lactose }\end{array} & \begin{array}{r}\text { Protéines } \\
\text { Lactose } \\
\text { Moyenne }\end{array} \\
\end{array}$ & $\begin{array}{l}50 \\
35\end{array}$ & 4,6 & 15,0 & $\begin{array}{l}24,8 \\
25,1 \\
25,0\end{array}$ & $\begin{array}{l}4,0 \\
5,3 \\
4,2 \\
\end{array}$ \\
\hline $\begin{array}{c}\text { Protéines } \\
\text { Lactosérum Lactose } \\
\text { Moyenne * }\end{array}$ & $\begin{array}{l}35 \\
65\end{array}$ & 3,1 & 12,3 & $\begin{array}{l}21,4 \\
19,6 \\
20,5\end{array}$ & $\begin{array}{l}6,7 \\
4,0 \\
5,0\end{array}$ \\
\hline
\end{tabular}

Théorique ${ }^{*}=$ valeur calculée par la théorie de VAN Rossum (1958).

Moyenne ${ }^{*}=$ moyenne calculée à partir des différents composants.

$\mathrm{M} \quad=$ moyenne des trois essais effectués avec chaque souillure.

E.T.M. = écart type de la moyenne des trois essais.

$\mathrm{C}_{\mathrm{o}} \quad=$ concentration initiale de la souillure.

$\gamma=$ viscosité cinématique de la souillure. 
façon que le volume d'effluent soit proportionnel à la durée de rinçage. Chaque type de souillure est testé trois fois.

\section{Caractérisation des solutions et produits utilisés}

Nous avons étudié l'aptitude au rinçage des souillures suivantes:

- de la soude et de l'acide nitrique (composants classiques des solutions de nettoyage), du chlorure et du fluorescéinate de sodium (traceurs utilisés par l'équipe de LoNCIN) et du saccharose. Ces différents produits simples ont été étudiés en solution à différentes concentrations, seuls ou en mélange ;

- du lait cru, entier, non homogénéisé ;

— du lait stérilisé U.H.T., entier, homogénéisé ;

- d'une solution de lactosérum doux de fromagerie concentrée par ultrafiltration puis évaporation et séchée par atomisation (Prodiest 351 de « PROSPÉRITÉ FERMIËRE »). Ce produit contient du lactose, de traces de minéraux et de protéines de sérum;

- d'une solution de lactose et de caséine « MERCK » isolée selon la technique de préparation d'HAMMARSTEN.

La composition et la teneur de ces différentes solutions sont précisées dans le tableau 1.

\section{Méthodes analytiques}

La matière grasse du lait a été dosée par absorbance (TISSIER et CORRIEU, 1983). Les protéines sont dosées par la méthode de Lowry et al. (1951), le saccharose et le lactose avec de l'anthrone par la méthode de Loewus (1952), le fluorescéinate de sodium par fluorimétrie, l'acide nitrique, la soude et le chlorure de sodium par conductimétrie.

\section{E. Mode d'expression des résultats}

La concentration en souillure de l'effluent est exprimée en concentration réduite $\left(C / C_{0}\right)$ qui est égale au rapport entre la concentration de l'effluent $(C)$ et la concentration initiale $\left(\mathrm{C}_{\mathrm{o}}\right)$ de la souillure.

La quantité totale de souillure présente sur la plaque après égouttage ne peut pas être déterminée expérimentalement. Il est néanmoins possible de calculer théoriquement l'épaisseur moyenne de film de souillure restant sur la plaque après égouttage $\left(\mathrm{e}_{\mathrm{o}}\right)$ avec la théorie de VAN Rossum (1958) qui prend en compte la viscosité cinématique de la souillure $(\gamma)$, la durée d'égouttage $(t)$ de la hauteur de la plaque (h),

$$
\mathrm{e}_{\mathrm{o}}=0,67(\Gamma \mathrm{h} / \mathrm{gt})^{1 / 2}
$$

La quantité de souillure éliminée par le rinçage est déterminée en multipliant le volume total d'effluent de rinçage par sa concentration relative en souillure. Le résultat est exprimé en volume de souillure initiale porté par unité de surface souil- 
lée de la plaque $\left(\mathrm{cm}^{3} \cdot \mathrm{m}^{-2}\right)$, qui correspond à une épaisseur équivalente de souillure initiale exprimée en micromètres.

La durée du rinçage est exprimée en temps réduit $\left(\mathrm{t}^{*}\right)$ qui est égal au rapport entre la durée du rinçage (t) et le temps de séjour moyen du film tombant sur la surface souillé $\left(\mathrm{t}_{5}\right)$.

\section{Résultats}

\section{A. Quantité de souillure éliminée par rinçage de la plaque}

Les résultats présentés dans le tableau 1 montrent que :

- Les quantités de souillure éliminées par le rinçage apparaissent très reproductibles car l'écart type correspondant à la moyenne des trois essais effectués avec chaque type de souillure est faible.

- L'estimation de la quantité moyenne de souillure présente sur la plaque après égouttage et éliminée par le rinçage ne dépend pas significativement du composé analysé. Ce résultat est valable pour les mélanges 1 et 2 et les produits laitiers. Il en résulte que la composition du film de souillure éliminé de la surface de la plaque par rinçage est identique à celle de la solution de souillure utilisée.

- La quantité de souillure demeurant sur la plaque après égouttage dépend de la souillure utilisée. Cette quantité varie avec la concentration de la souillure (un sirop de saccharose à 480 g. $1^{-1}$ laisse un film 7 fois plus important qu'un sirop de saccharose à $150 \mathrm{~g} . \mathrm{l}^{-1}$ ) et avec la nature de la souillure (les solutions de chlorure de sodium à $20 \mathrm{~g} . \mathrm{l}^{-1}$, d'acide nitrique à $14 \mathrm{~g} . \mathrm{l}^{-1}$ et de soude à $20 \mathrm{~g} . \mathrm{l}^{-1}$ laissent sur la paroi après 35 minutes d'égouttage, une quantité de souillure respectivement égale à environ 3,5 et $11 \mathrm{~cm}^{3} \cdot \mathrm{m}^{-2}$ ). Pour les produits laitiers, la quantité moyenne de souillure laissée par la solution de caséine soluble ou le lactosérum est plus importante que celle laissée par le lait cru entier et le lait stérilisé U.H.T. entier homogénéisé. Cette augmentation est probablement corrélée à la formation de mousses plus ou moins abondantes pendant la souillure de la plaque, mousses qui ne sont que partiellement éliminées pendant l'égouttage.

\section{B. Cinétique de rinçage de composés simples}

Nous présentons à la figure 2 , pour le fluorescéinate de sodium à $0,2 \mathrm{~g} \cdot \mathrm{l}^{-1}$, dans les mélanges $1(\mathrm{x})$ et 2 (o), l'évolution pendant le rinçage de la concentration réduite en souillure de l'effluent $\left(C / C_{0}\right)$ en fonction du temps réduit $\left(t^{*}\right)$ ou de l'épaisseur équivalente de souillure restant sur la plaque $\left(\mathrm{e}_{\mathrm{s}}\right)$. Ces variables sont présentées dans des échelles normales et (ou) logarithmiques.

La durée de rinçage nécessaire pour abaisser la concentration réduite en souillure de l'effluent à une valeur donnée est fortement influencée par $\mathrm{e}_{\mathrm{o}}$, épaisseur initiale de souillure présente sur la plaque (fig. $2 \mathrm{~A}, 2 \mathrm{C}$ ). La concentration réduite en souillure de l'effluent ne semble dépendre que de l'épaisseur équivalente $e_{s}$ de souillure restant sur la plaque à un instant donné et $\mathrm{e}_{\mathrm{o}} \mathrm{n}$ 'influence pas ce résultat (fig. $2 \mathrm{~B}$ et $2 \mathrm{D}$ ). Le même phénomène est observable avec le chlorure de sodium et le saccharose. 

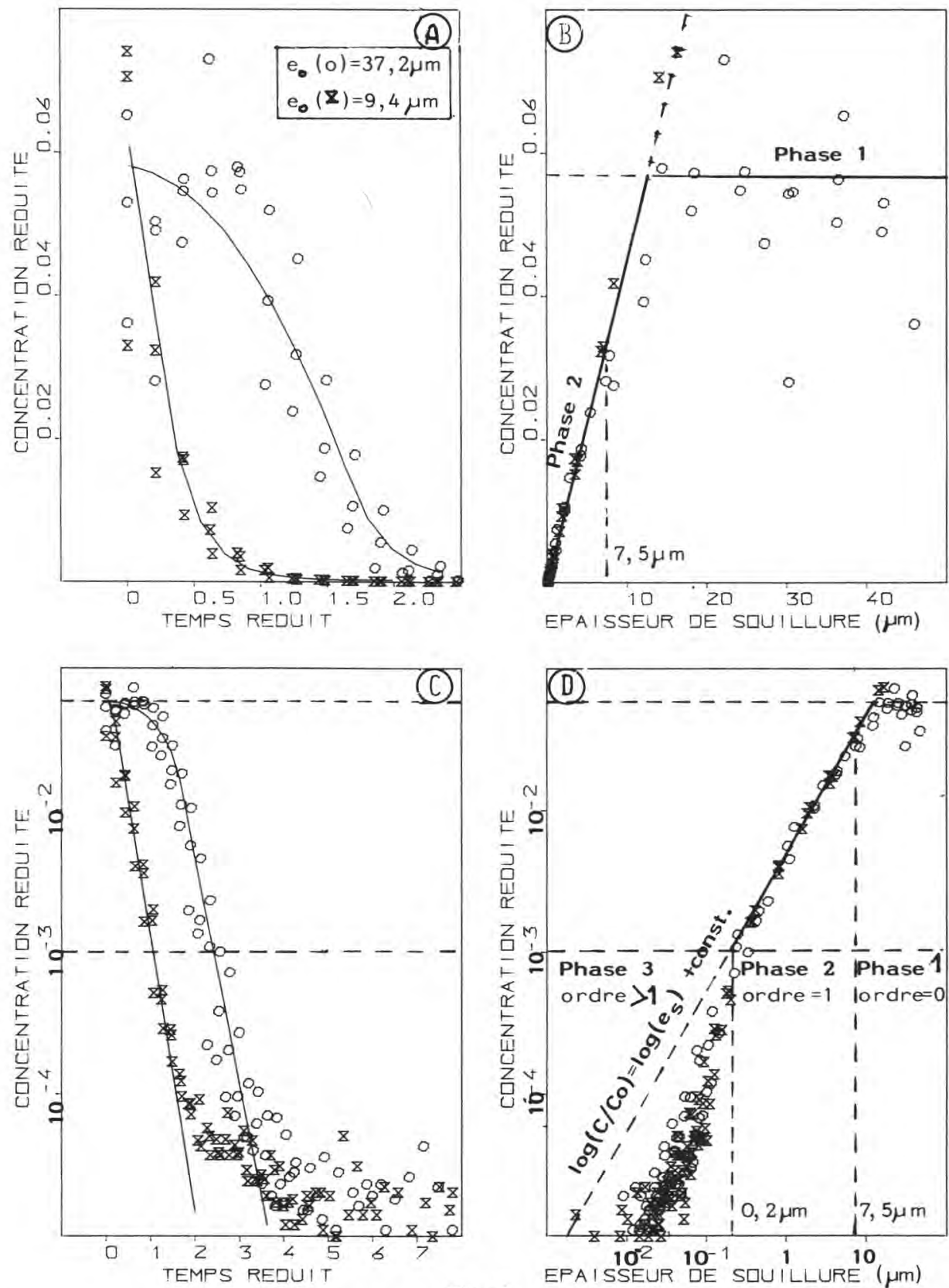

Fig. 2

Evolution de la concentration en fluorescéinate de sodium de l'effluent en fonction du temps et de l'épaisseur équivalente de souillure restant à la paroi, pendant le rinçage d'une souillure comprenant $0,2 \mathrm{~g} / \mathrm{l}$ de fluorescéinate et $100 \mathrm{~g} / \mathrm{l}$ de $\mathrm{NaCl}$ (mélange 1 ( $\mathrm{X}$ ) ou 0,2 $\mathrm{g} / \mathrm{l}$ de fluorescéinate, $100 \mathrm{~g} / \mathrm{l}$ de $\mathrm{NaCl}$ et $480 \mathrm{~g} / \mathrm{l}$ de saccharose (mélange 2 (0)).

La cinétique des deux premières phases du rinçage simulée par le modèle prenant en compte la répartition de la souillure le long de la plaque est présentée sur les figures $2 A$ et $2 C$.

$\mathrm{Na}$-fluorescein concentration in the outflow as a fonction of time and equivalent soil thickness remaining on the wall during the rinsing of a soil containing $0,2 \mathrm{~g}^{-l^{-1}}$ of $\mathrm{Na}$-fluorescein and $100 \mathrm{~g} . \mathrm{l}^{-1} \mathrm{NaCl}$ (mixture 1 (X)) or $0,2 \mathrm{~g} . \mathrm{l}^{-1}$ of $\mathrm{Na}$-fluorescein, 100

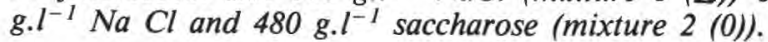

The kinetics of the two first rinsing phase calculated by the model taking into account the soil distribution along the plate is presented on figures $2 A$ and $2 C$. 
Il apparaît que le rinçage peut être traduit par trois phases qui s'enchaînent.

La première phase du rinçage ne peut être mise en évidence que lorsque $e_{0}$ est supérieure à une quinzaine de micromètres. Pendant cette phase $\left(C / C_{0}\right)$ reste relativement constant et ne dépend pas de $\mathrm{e}_{\mathrm{s}}$.

La deuxième phase du rinçage correspond à une décroissance exponentielle rapide de $\left(\mathrm{C} / \mathrm{C}_{\mathrm{o}}\right)$ en fonction du temps (fig. 2B). Cette phase assure l'élimination de souillure dont l'épaisseur équivalente est comprise entre 7,5 et $0,2 \mu \mathrm{m}$. $\left(\mathrm{C} / \mathrm{C}_{\mathrm{o}}\right)$ varie proportionnellement à $\mathrm{e}_{\mathrm{s}}$ (fig. 2B).

\section{TABLeAu 2}

Paramètres caractérisant la souillure et la cinétique de rinçage de l'ensemble des composés simples. Résultats exprimés par rapport au temps réduit et la concentration réduite.

Characteristics and rinsing kinetics parameters of simple compound soiling. Results are expressed in dimensionless concentration and time.

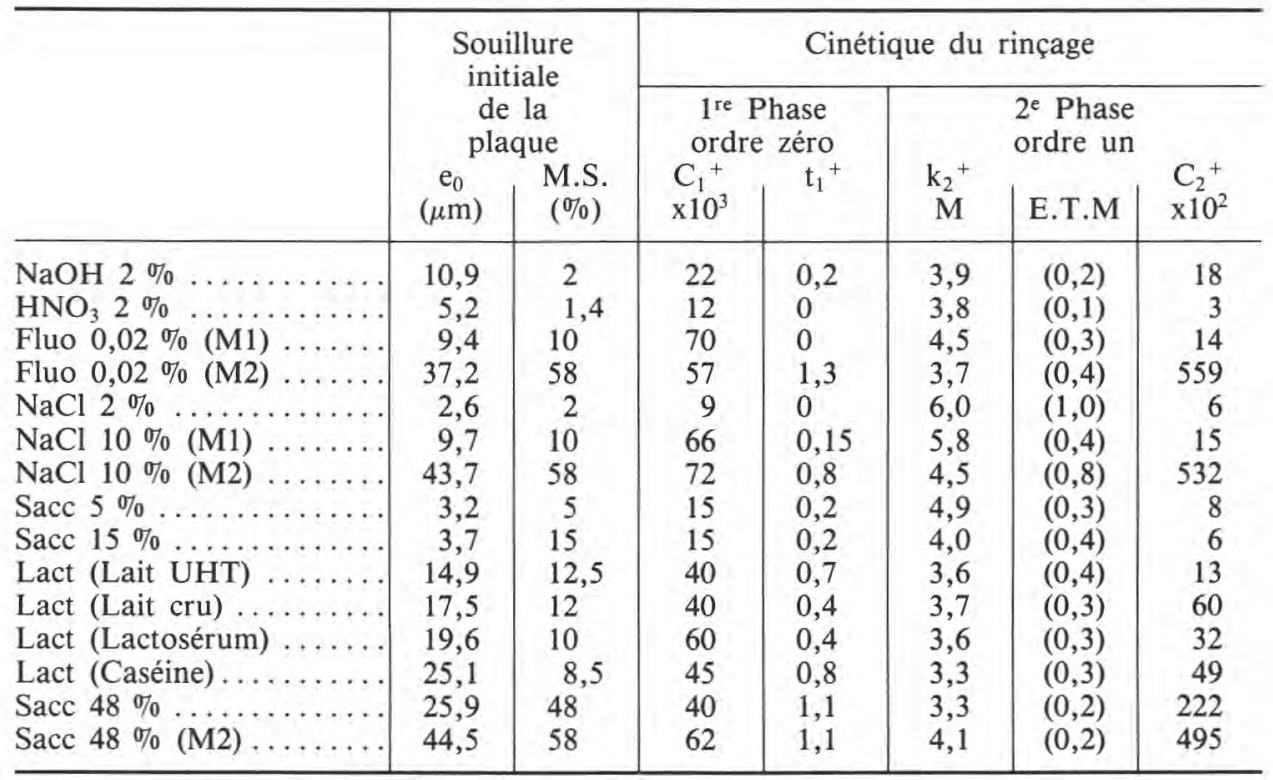

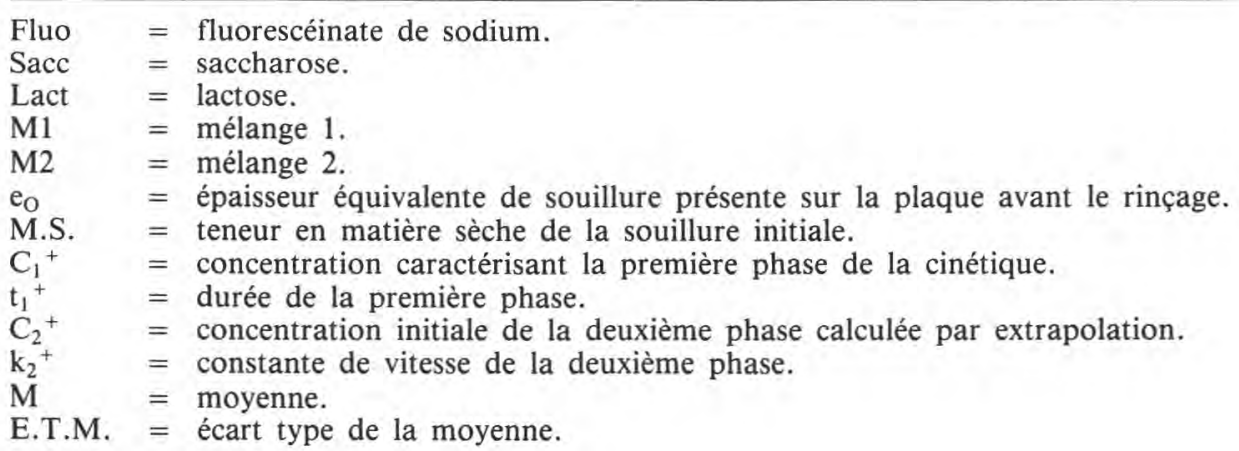



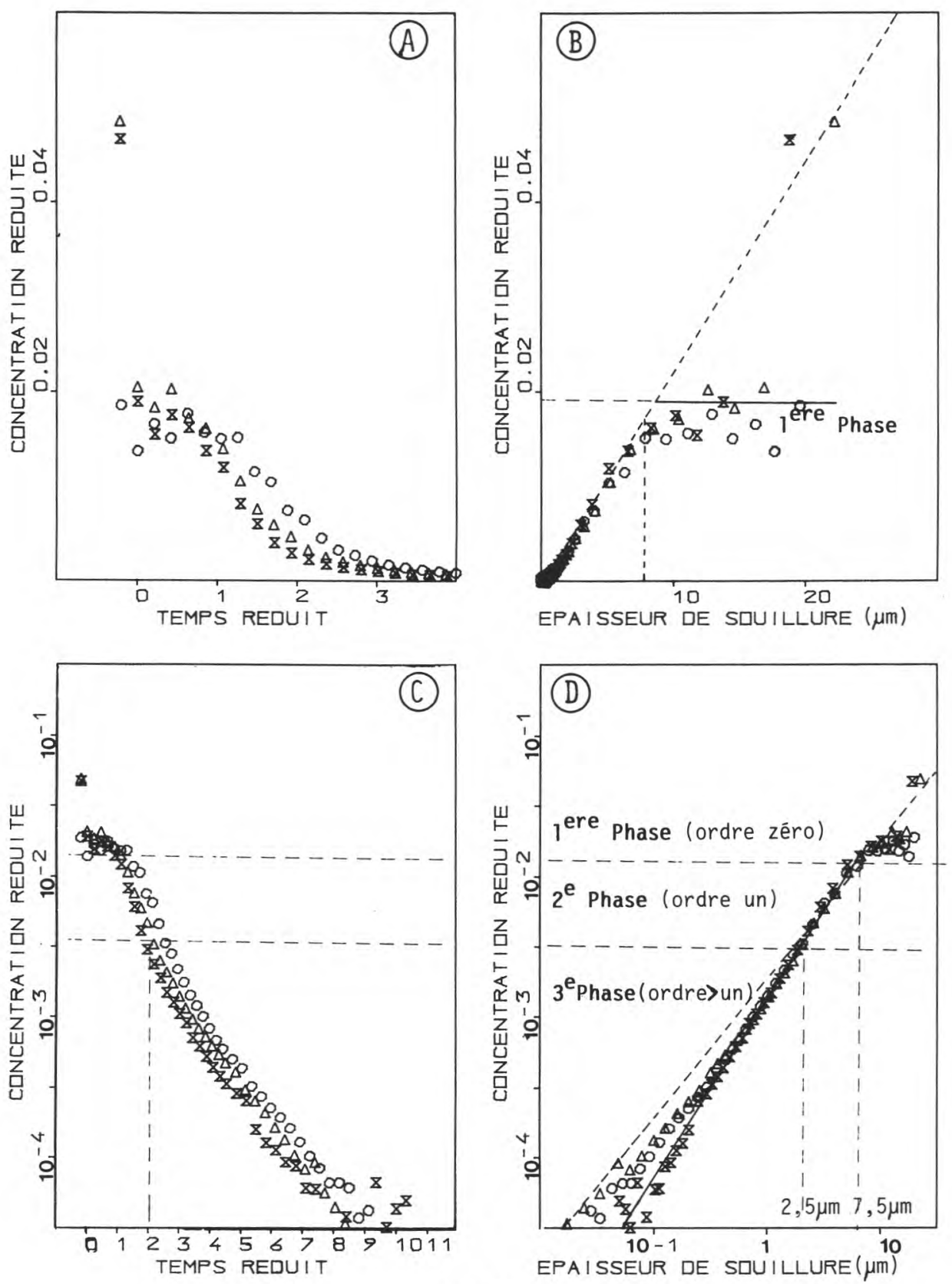

Fig. 3

Evolution de la concentration réduite en protéines de l'effluent en fonction du temps et de l'épaisseur équivalente en protéines de souillure restant à la paroi, pendant le rinçage d'une souillure constituée de lait entier cru, non homogénéisé.

Protein concentration in the outflow as a fonction of time and equivalent soil thickness remaining on the wall during the rinsing of a soil composed of non homogenized whole raw milk. 
Paramètres caractérisant la souillure et la cinétique de rinçage de la matière grasse et des protéines des produits laitiers.

Résultats exprimés par rapport au temps réduit de la concentration réduite.

Soil and rinsing kinetics parameters characterizing fat and proteins of dairy products. Results are expressed in dimensionless concentration and time.

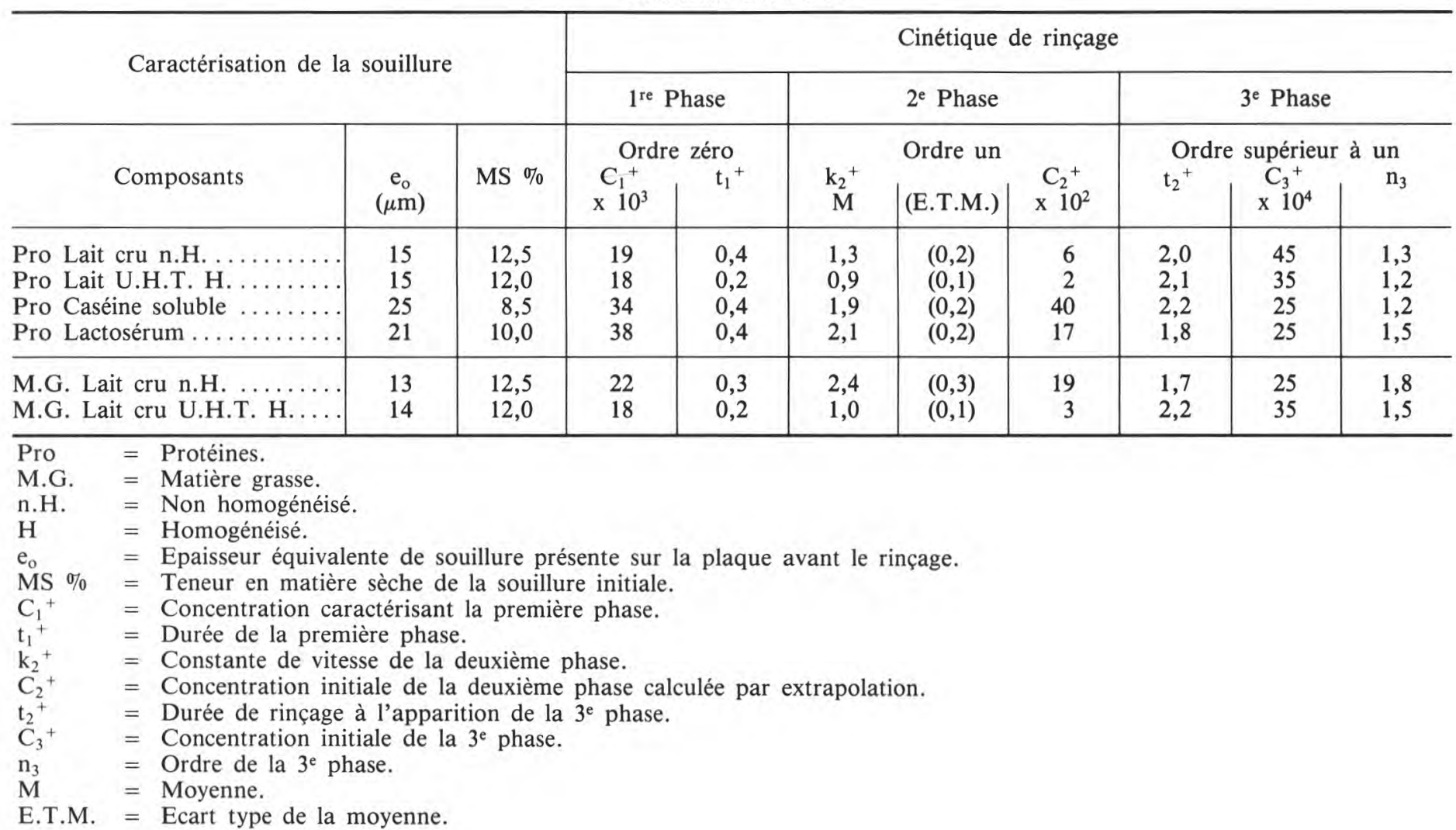


La troisième phase de rinçage est caractérisée par un ralentissement de la décroissance exponentielle de la concentration en souillure de l'effluent en fonction du temps. Ce phénomène n'est observable que lorsque l'épaisseur équivalente de souillure restant sur la plaque est faible (inférieure à 0,2 micromètre). La concentration réduite en souillure de l'effluent correspondant à ce seuil $\left(\mathrm{C}_{3}{ }^{+}\right)$est voisine de $10^{-3}$.

Remarque : Le lactose, composé simple entrant dans la composition des quatre produits laitiers étudiés, et son isomère chimique le saccharose de diffusivité à $25^{\circ} \mathrm{C}$ égale à $4,9.10^{-10} \mathrm{~m}^{2} \cdot \mathrm{s}^{-1}$ (Liley et Gambill, 1973), qui présentent un comportement identique, ont été regroupés dans le tableau 2.

\section{Cinétique de rinçage des produits laitiers}

Les trois phases caractéristiques du rinçage des composés simples sont également observables lors du rinçage des protéines et de la matière grasse des produits laitiers. L'importance relative de ces différentes phases est cependant très différente comme le montrent par exemple la figure 3 relative aux protéines du lait cru et le tableau 3 caractérisant la cinétique de rinçage des différents produits laitiers.

La première phase qui assure l'élimination des fortes épaisseurs équivalentes de souillures est courte car l'épaisseur initiale de souillure portée par la plaque reste limitée (entre 13 et 25 micromètres).

La deuxième phase du rinçage est nettement plus courte que celle observée lors du rinçage des composés simples. Cette phase assure seulement l'élimination de souillures dont l'épaisseur équivalente est comprise entre 7,5 et 2,5 micromètres (protéines du lait cru, protéines du lait stérilisé U.H.T. et matière grasse du lait homogénéisé) ou en 7,5 et 0,8 micromètres (protéines du lactosérum, caséine soluble, matière grasse du lait non homogénéisé).

La troisième phase du rinçage qui assure l'élimination de quantités relativement importantes de souillure ne peut plus être négligée.

\section{Discussion}

\section{A. Quantité de souillure éliminée par rinçage de la plaque}

Le tableau 1 montre que la quantité de souillure présente sur la plaque après 35 minutes d'égouttage, calculée avec la théorie de VAN Rossum (1958) est assez bien corrélée à la quantité de souillure éliminée par le rinçage qui d'après nos précédents résultats (Tissier et al., 1983a) assure l'élimination de 90 à $100 \%$ de la souillure présente sur la paroi.

Cependant, la quantité du mélange 2 (contenant $480 \mathrm{~g} . \mathrm{l}^{-1}$ de saccharose, $100 \mathrm{~g} .1^{-1}$ de $\mathrm{NaCl}$ et $0,2 \mathrm{~g} . \mathrm{l}^{-1}$ de fluorescéinate de sodium) présente sur la plaque verticale est nettement plus importante que celle calculée théoriquement par mesure de la viscosité de cette solution de souillure. Nous avons discuté plusieurs hypothèses (TISSIER, 1984) pour expliquer ce phénomène. Il est notamment probable que le film de souillure dont la concentration est très élevée et qui s'écoule 
très lentement se concentre par évaporation sur la plaque ; il en résulterait soit une cristallisation, soit une augmentation importante de la viscosité du film tombant. Il faut par ailleurs rappeler que la relation de VAN Rossum (1958) suppose que l'influence de la tension superficielle est négligeable. Cette dernière hypothèse peut, vu les très fortes teneurs en matières sèches observées, ne plus être valable.

\section{B. Cinétique de rinçage des composés simples et des produits laitiers}

Le rinçage comprend trois phases qui s'enchaînent. La première phase du rinçage peut être traduite par un modèle d'ordre zéro puisque pendant cette phase, la concentration réduite en souillure de l'effluent $\left(C / C_{o}\right)$ reste relativement constante et ne dépend pas de l'épaisseur équivalente de souillure restant sur la plaque $\left(\mathrm{e}_{\mathrm{s}}\right)$. Les tableaux 2 et 3 précisent la valeur des deux paramètres qui caractérisent cette phase : la concentration relative en souillure de l'effluent de rinçage $\mathrm{C}_{1}{ }^{+}$et la durée $\mathrm{t}_{1}{ }^{+}$exprimée en temps réduit.

Lorsque la première phase du rinçage n'est pas mise en évidence, la valeur de la concentration $\mathrm{C}_{1}+$ indiquée dans le tableau 2 correspond à la valeur initiale de la concentration réduite en souillure de l'effluent. Il apparaît que :

$-\mathrm{C}_{1}{ }^{+}$dépend de la nature de la souillure et du composé considéré.

- La durée de cette première phase $\mathrm{t}_{1}{ }^{+}$est d'autant plus grande que $\mathrm{C}_{1}{ }^{+}$est faible et que l'épaisseur équivalente de souillure portée par la plaque avant le rinçage est forte. Cependant, l'estimation de cette durée est peu précise car l'origine des temps n'est pas connue avec précision et la séparation entre les deux premières phases de la cinétique n'est pas nette.

La deuxième phase du rinçage peut être traduite par un modèle du premier ordre puisque la concentration réduite en souillure de l'effluent $\left(C / C_{0}\right)$ suit une décroissance exponentielle en fonction du temps. Les tableaux 2 et 3 précisent la valeur des deux paramètres qui définissent cette deuxième phase : la constante de vitesse $\mathrm{k}_{2}{ }^{+}$et la concentration initiale en souillure de l'effluent $\left(\mathrm{C}_{2}{ }^{+}\right)$, calculée par extrapolation à l'origine des temps, du logarithme de la concentration en souillure de l'effluent.

Il apparaît que :

- Pour une souillure donnée, $\mathrm{k}_{2}{ }^{+}$qui dépend de la nature du composé considéré est très reproductible.

$-\mathrm{k}_{2}{ }^{+}$est nettement plus élevée pour les composés simples que pour les protéines et la matière grasse du lait.

$-\mathrm{k}_{2}{ }^{+}$est environ deux fois plus faible pour les protéines du lait et la matière grasse du lait homogénéisé que pour les protéines du lactosérum, la caséine soluble et la matière grasse du lait non homogénéisé. Pour les souillures contenant du chlorure de sodium ou des sucres (saccharose et lactose) dont l'épaisseur initiale de souillure avant le rinçage $\left(e_{o}\right)$ varie sur une grande plage, une augmentation de $\mathrm{e}_{\mathrm{o}}$ (ou de la viscosité) entraîne une diminution de $\mathrm{k}_{2}{ }^{+}$.

- Dans le cas d'un mélange, $\mathrm{k}_{2}{ }^{+}$dépend de la nature du composant considéré, mais un phénomène d'intéraction se produit qui tend à rapprocher le comportement des différents composants. 
- Pour l'ensemble des cinétiques, le logarithme de $\mathrm{C}_{2}{ }^{+} / \mathrm{k}_{2}{ }^{+}$semble varier linéairement avec $\mathrm{e}_{\mathrm{o}}$.

- La valeur de $\mathrm{C}_{1}{ }^{+}$, caractérisant la première phase du rinçage, contrôlée par un modèle réactionnel d'ordre zéro dépend de $\mathrm{e}_{0}$. L'utilisation du rapport $\mathrm{C}_{1}{ }^{+} / \mathrm{k}_{2}{ }^{+}$permet de réduire la variabilité des résultats. Pour les faibles épaisseurs initiales de souillure, le rapport $\mathrm{C}_{1}{ }^{+} / \mathrm{k}_{2}{ }^{+}$augmente linéairement avec $\mathrm{e}_{\mathrm{o}}$. Lorsque la première phase du rinçage qui suit un modèle réactionnel d'ordre zéro peut être mise en évidence, c'est-à-dire lorsque $e_{0}$ est supérieur à une quinzaine de micromètres, le rapport $\mathrm{C}_{1}{ }^{+} / \mathrm{k}_{2}{ }^{+}$semble relativement constant. Ces propriétés des rapports $\mathrm{C}_{1}{ }^{+} / \mathrm{k}_{2}{ }^{+}$et $\mathrm{C}_{2}{ }^{+} / \mathrm{k}_{2}{ }^{+}$permettent de paramétrer le modèle qui traduit la cinétique du rinçage.

La troisième phase du rinçage est caractérisée par un ralentissement de la décroissance exponentielle de $\mathrm{C} / \mathrm{C}_{0}$ en fonction du temps. Cette dernière phase qui dépend fortement de la nature de la souillure peut être traduite empiriquement par un modèle réactionnel d'ordre $\mathrm{n}_{3}$, supérieur à un, dont la valeur est déterminée sur le tracé du logarithme de $\mathrm{C} / \mathrm{C}_{\mathrm{o}}$ en fonction du logarithme de $\mathrm{e}_{\mathrm{s}}$ à partir de la pente de la droite reliant les points expérimentaux de cette phase.

Pour les composés simples, ce ralentissement n'étant pas mis en évidence avec des souillures dont la teneur en matière sèche est faible, il est probable que les forces de viscosité influent sur l'élimination de très faibles épaisseurs équivalentes de souillure résiduelle.

La transition entre la deuxième et la troisième phase de la cinétique s'effectue de façon marquée. Elle correspond à une même valeur $(0,2 \mu \mathrm{m}$ de souillure résiduelle) si le résultat est exprimé en épaisseur équivalente de souillure et non en masse de composant. Nous supposons donc que cette dernière phase correspond à l'élimination de volumes de souillure piégée dans les microrugosités profondes de la plaque.

En utilisant une approche semblable à celle de SCHLUSSLER et KocH (1979), il est possible de calculer qu'une épaisseur équivalente de souillure de 0,2 micromètre présente sur la surface d'un réservoir de $2,5 \mathrm{~m}^{3}$ entraîne une dilution de cette souillure de plus de un million de fois lors du remplissage du réservoir. Cette dilution augmente avec la capacité du réservoir. Aussi, avec un certain nombre de produits, en particulier avec la soude ou l'acide nitrique, une telle dilution dans un produit alimentaire est indécelable et ne présente aucun danger pour le consommateur. En conséquence, il pourrait être envisagé, dans certains cas, de limiter la pratique du rinçage aux deux premières phase du processus.

Pour les protéines et la matière grasse du lait, la troisième phase du rinçage qui assure l'élimination d'une quantité non négligeable de souillure $(0,8$ ou $2,5 \mu \mathrm{m}$ d'épaisseur équivalente) doit être prise en considération. Nous avons présenté dans le tableau 3, les trois paramètres qui caractérisent cette troisième phase : la durée de rinçage $\left(\mathrm{t}_{2}{ }^{+}\right)$, la concentration en souillure de l'effluent correspondant en début de cette troisième phase $\left(\mathrm{C}_{3}{ }^{+}\right)$et l'ordre du modèle réactionnel $\left(\mathrm{n}_{3}\right)$.

\section{Interprétation des résultats, mécanismes contrôlant le rinçage}

L'ensemble des résultats montre que l'épaisseur équivalente de souillure correspondant à la transition entre les deux premières phases de la cinétique est égale 
à 7,5 micromètres et ne dépend pas de la nature du composé considéré. Ces observations conduisent à supposer qu'un même mécanisme contrôle les deux premières phases du rinçage et que l'élimination d'un composé dépend de son aptitude au rinçage, aptitude qui est quantifiable à l'aide de $\mathrm{k}_{2}{ }^{+}$.

Nous allons étudier si un mécanisme de diffusion moléculaire permet d'expliquer la différence de comportement des différentes souillures pendant le rinçage.

Pour les électrolytes, le coefficient de diffusivité à $25^{\circ} \mathrm{C}$ du chlorure de sodium, de l'hydroxyde de sodium et de l'acide nitrique qui est respectivement égal à $15.10^{-10}, 21.10^{-10}$ et $30.10^{-10} \mathrm{~m}^{2} \cdot \mathrm{s}^{-1}$ n'est pas corrélé à l'aptitude au rinçage de ces produits. En effet, comme le montre le tableau 2, la constante de vitesse $\mathrm{k}_{2}{ }^{+}$exprimée par unité de temps de séjour, est respectivement égale à 6,0 , 3,9 et 3,8 .

Le coefficient de diffusivité des protéines du lactosérum et de la caséine est évalué respectivement à $0,5 \cdot 10^{-10}$ et $0,05 \cdot 10^{-10} \mathrm{~m}^{2} \cdot \mathrm{s}^{-1}$ (KessLeR, 1981). Les micelles de caséine sont constituées de sous unités sphériques de 15 à 20 nanomètres de diamètre dont la masse moléculaire selon SCHMIDT (1980) est voisine de 250000 . Il est probable que le traitement de préparation de la caséine soluble " MercK " brise la structure des micelles de caséine, mais que ce traitement conserve intacte la structure des sous-unités sphériques dont la diffusivité peut être évaluée à $0,3 \cdot 10^{-10} \mathrm{~m}^{2} \cdot \mathrm{s}^{-1}$, en utilisant l'équation de StOKES-EINSTEIN (ATKins, 1982). Cette même équation conduit à calculer en coefficient de diffusivité respectivement égal à $0,001 \cdot 10^{-10} \mathrm{~m}^{2} \cdot \mathrm{s}^{-1}$ et à $0,004 \cdot 10^{-10} \mathrm{~m}^{2} \cdot \mathrm{s}^{-1}$ pour la matière grasse du lait non homogénéisé et du lait homogénéisé en supposant le diamètre des globules gras respectivement égal à $4 \mu \mathrm{m}$ et à $1 \mu \mathrm{m}$.

Le comportement des différents composés étudiés ne dépend donc pas uniquement de leur diffusivité. Il apparaît, néanmoins, que les petites molécules $(\mathrm{NaCl}$, saccharose...) dont la diffusivité est élevée sont éliminées plus rapidement que les protéines et la matière grasse du lait. De même les protéines de faible taille moléculaire (protéines du lactosérum, caséine soluble) dont la diffusivité est plus élevée sont éliminées plus rapidement que les micelles de caséine dont la taille est plus élevée. L'influence de l'homogénéisation sur les globules gras qui n'est pas conforme avec l'ensemble des résultats peut être expliquée au moins partiellement : la taille des globules gras du lait non homogénéisé, généralement comprise entre 2 et 12 micromètres, est le plus souvent supérieure à la dimension des rugosités de la plaque $(3,5$ micromètres en moyenne si on considère la distance entre les crêtes et les creux). Les globules gras du lait non homogénéisé peuvent donc être plus facilement entraînés par le film tombant lors du rinçage.

Cependant, étant donné que la valeur des coefficients de diffusivité des composants étudiés varie sur une plage de 1 à 30000 alors que la valeur de la constante $\mathrm{k}_{2}{ }^{+}$ne varie que sur une plage de 1 à 7 , un simple mécanisme de diffusion pure ne permet pas d'expliquer la deuxième phase de la cinétique de rinçage.

La troisième phase du rinçage se manifeste lorsque l'épaisseur équivalente de souillure est inférieure à 2,5 micromètres pour les protéines du lait cru et du lait stérilisé U.H.T. Compte tenu de la concentration initiale en protéines des solutions de souillure, un calcul montre que l'épaisseur équivalente de protéines adhérant à la paroi pourrait être de l'ordre de 90 nanomètres, ce qui correspond sensiblement à une couche moléculaire de micelles de caséine. Il est possible de faire 
le même raisonnement pour les globules gras du lait U.H.T. homogénéisé. Pour les protéines du lactosérum et la caséine soluble, l'épaisseur équivalente de protéine correspondant à la transition entre la deuxième et la troisième phase de la cinétique est voisine de 30 nanomètres. L'épaisseur équivalente de caséine restant à la paroi au moment de la transition entre la deuxième et la troisième phase de la cinétique peut donc correspondre à une ou deux couches de sous-unités de caséine. Un raisonnement voisin (TISSIER, 1984) conduit à supposer que l'épaisseur de protéines du lactosérum résiduelles correspond à seulement quelques couches moléculaires lorsque la troisième phase du rinçage commence à se manifester.

Il en résulte que, pour les protéines et la matière grasse du lait homogénéisé, la transition entre la deuxième et la troisième phase du rinçage apparaît lorsqu'une épaisseur de l'ordre de une ou de quelques molécules ou unités de composant reste à la paroi. Cette troisième phase est donc compatible avec un mécanisme de désorption de macromolécules portant des charges électriques (protéines ou matière grasse), ayant préalablement adhéré à la paroi. Ceci est en accord avec les travaux effectués sur des canalisations par PLETT (1984).

Pour les composés simples, nous avons vu au paragraphe III.B que la troisième phase de la cinétique de rinçage correspond à l'élimination de souillure «piégée » dans des microrugosités profondes de la paroi.

Il semble donc que la troisième phase de la cinétique de rinçage soit contrôlée par des phénomènes de surface que notre dispositif expérimental ne permet pas d'étudier de façon précise.

\section{D. Établissement du modèle}

La discussion de la mise en équation des transferts de matière permettant l'établissement du modèle a été détaillée par ailleurs (TISSIER, 1984). Nous présentons seulement dans ce texte une synthèse de ce travail. Le modèle retenu traduit les deux premières phases du rinçage et leur enchaînement en prenant en compte les aspérités microscopiques de la surface qui sont supposées former des cavités identiques de profondeur $e_{p}$. Le volume de souillure porté par unité de surface de la plaque permet de définir une épaisseur équivalente de souillure $e_{s}$ qui peut être supérieure, inférieure ou égale à la profondeur équivalente $\mathrm{e}_{\mathrm{p}}$ des cavités.

La surface souillée, d'aire S, est rincée au débit Q par le film tombant. L'élimination de la souillure s'effectue à travers une couche limite d'épaisseur $e_{1}$, négligeable devant l'épaisseur e du film tombant, selon un mécanisme diffusionnel qui est régi par la première loi de Fick : l'interface entre la couche limite et le film tombant de rinçage étant en équilibre avec le film tombant, sa concentration est égale à la concentration en souillure $C_{f}$ du film tombant. De même, nous supposons que la concentration de l'interface entre la couche limite et la souillure est égale à la concentration de la souillure $\mathrm{C}_{\mathrm{s}}$.

La première phase du rinçage schématisée à la figure $4 \mathrm{~A}$ concerne l'élimination de souillures dont l'épaisseur $\mathrm{e}_{\mathrm{s}}$ est supérieure à la profondeur $\mathrm{e}_{\mathrm{p}}$ des cavités. Comme les résultats présentés au tableau 1 montrent que ces conditions opératoires ne sont observables que lorsque la viscosité de la solution de souillure est élevée, nous supposons que le film de souillure est immobile et que le film de rinçage 


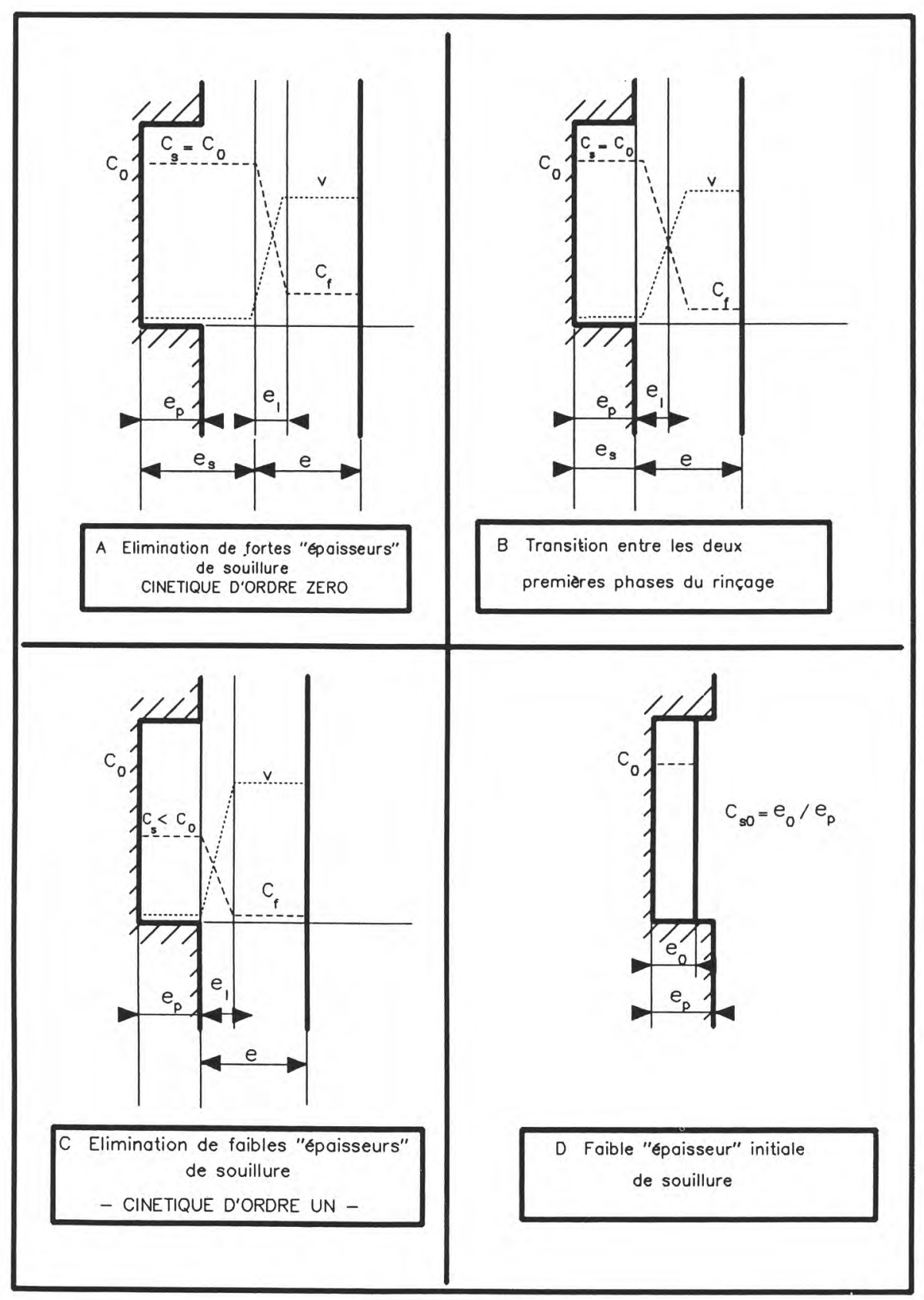

Fig. 4

Schéma indiquant les caractéristiques du modèle permettant de simuler le rinçage d'une surface par un film tombant (voir la nomenclature pour connaître la signification des paramètres).

Model scheme used to simulate the rinsing of a flat plate by a falling film (see notation to understand variables). 
qui s'écoule en régime turbulent glisse sur le film de souillure sans le pénétrer et donc sans mélange. Le rinçage entraîne donc une diminution de l'épaisseur de la souillure dont la concentration reste égale à la concentration initiale $\mathrm{C}_{\mathrm{o}}$. Le bilan de matière et l'utilisation de la première loi de FICK (relation 3 ) permettent de montrer que la concentration en souillure de l'effluent $C_{1}{ }^{*}$ reste constante pendant cette phase (relation 4) si on admet que la concentration en souillure du film tombant $\left(\mathrm{C}_{\mathrm{f}}\right)$ est négligeable par rapport à la concentration initiale de la souillure $\left(\mathrm{C}_{\mathrm{o}}\right)$.

$$
\begin{gathered}
C Q d t=-C_{0} S d e_{s}=D\left[\left(C_{o}-C_{f}\right) / e_{l}\right] S d t \approx\left(D / e_{1}\right) C_{o} S d t \\
C_{1} *=C / C_{0} \approx D S / e_{1} Q=D t_{s} / e_{1} e
\end{gathered}
$$

La deuxième phase du rinçage apparaît lorsque, par suite du rinçage, l'épaisseur résiduelle de souillure $e_{s}$ devient égale à la profondeur de la cavité $\mathrm{e}_{\mathrm{p}}$. Une réaction d'ordre un s'enchaîne alors à la réaction d'ordre zéro. Cette transition est schématisée à la figure 4B.

Nous supposons alors, selon le modèle schématisé à la figure $4 \mathrm{C}$, que le film tombant turbulent de rinçage ne pénètre pas les cavités, dont la concentration en souillure $C_{s}$ reste homogène et que la concentration de l'interface entre la couchè limite et les cavités est égale à $C_{s}$. Le rinçage produit une diminution de la concentration en souillure des cavités qui est calculable à partir du bilan de matière et de la première loi de FICK. En négligeant la concentration du film tombant $C_{f}$ par rapport à la concentration des cavités $C_{s}$, nous obtenons la relation 5 qui montre que l'élimination de la souillure pendant cette phase suit un modèle réactionnel d'ordre un. La relation 6 montre que la constante de vitesse $\mathrm{k}_{2}{ }^{+}$de cette deuxième phase, exprimée par unité de temps de séjour est lié à $\mathrm{C}_{1}{ }^{+}$, concentration en souillure de l'effluent pendant la première phase du rinçage.

$$
\begin{gathered}
C Q d t=-e_{p} S d_{s}=D\left[\left(C_{s}-C_{f}\right) / e_{1}\right] S d t \approx\left(D / e_{1}\right) C_{s} S d t \\
k_{2}{ }^{+}=-\left(D t_{s}\right) /\left(e_{l} e_{p}\right)=C_{1}+e / e_{p}
\end{gathered}
$$

Lorsque la viscosité de la solution de souillure est faible, l'épaisseur initiale de souillure est inférieure ả la profondeur des cavités. Lors de la mise en place du film tombant turbulent, il se produit une dilution instantanée de la souillure dans les cavités dont la concentration réduite initiale $\left(\mathrm{C}_{\mathrm{so}}{ }^{+}\right)$devient égale au rapport entre l'épaisseur initiale de souillure et la profondeur des cavités (fig. 4D). L'élimination de cette souillure est alors contrôlée par un modèle réactionnel d'ordre un comme précédemment.

Le modèle peut être appliqué en tenant compte de la répartition de l'épaisseur de souillure sur la plaque qui, selon VAN Rossum (1958), suit un profil parabolique.

Quatre paramètres permettent de définir le modèle. Certains correspondent à des données expérimentales comme l'épaisseur équivalente de souillure présente sur la plaque au début du rinçage $\left(\mathrm{e}_{0}\right)$ et l'épaisseur du film tombant de rinçage (e).

D'autres paramètres (le coefficient de transfert de matière de la souillure $\left(\mathrm{D} / \mathrm{e}_{1}\right) /\left(\mathrm{C}_{\mathrm{s}}-\mathrm{C}_{\mathrm{f}}\right)$ et la profondeur des cavités $\left.\left(\mathrm{e}_{\mathrm{p}}\right)\right)$ doivent être estimés à partir des résultats expérimentaux. 
Le modèle permet d'estimer la profondeur moyenne des rugosités en comparant la valeur des rapports $\mathrm{C}_{1}{ }^{+} / \mathrm{k}_{2}{ }^{+}$et $\mathrm{C}_{2}{ }^{+} / \mathrm{k}_{2}{ }^{+}$obtenus par la simulation, aux valeurs des mêmes rapports déterminées expérimentalement pour chacune des 62 cinétiques de rinçage. Pour l'ensemble des résultats, une profondeur moyenne de 8 micromètres semble traduire correctement le phénomène.

Ce résultat est à rapprocher des observations de DAUFIN (Communication personnelle) qui précise que la rugosité moyenne de la plaque est voisine de 3,5 micromètres. Le volume de souillure qui est éliminé selon une cinétique d'ordre un est grossièrement du même ordre de grandeur que le volume de souillure susceptible d'être inclus dans les rugosités de la plaque.

Ce résultat a permis de simuler correctement les deux premières phases du rinçage correspondant à chacun des composés étudiés, seuls ou en mélange, en prenant une profondeur de cavité égale à 8 micromètres et les épaisseurs initiales de souillure $\left(\mathrm{e}_{0}\right)$ et les constantes de vitesses $\left(\mathrm{k}_{2}{ }^{+}\right)$indiquées dans les tableaux 2 et 3 . Deux simulations sont possibles : une simulation complète présentée sur les figures $2 \mathrm{~A}$ et $2 \mathrm{C}$, pour le fluorescéinate de sodium qui prend en compte la répartition de la souillure le long de la plaque, et une simulation simplifiée qui ne tient pas compte de cette répartition. La différence entre les deux modèles est négligeable pour les faibles épaisseurs initiales de souillure. Lorsque l'épaisseur initiale de souillure devient importante, l'allure des cinétiques calculées avec la simulation complète semble plus proche des résultats expérimentaux car elle rend compte de l'évolution progressive de la concentration en souillure de l'effluent qui est observable entre les deux premières phases du rinçage.

\section{E. Validité du dispositif expérimental}

Afin de vérifier si notre dispositif expérimental permet de simuler un tank de stockage, la comparaison entre le rinçage de lait cru entier non homogénéisé présent sur la plaque et le rinçage du lait pasteurisé entier non homogénéisé, contenu dans un tank de stockage après égouttage a été détaillée par ailleurs (TISSIER, 1984).

Les principaux résultats obtenus montrent que :

- Les quantités de souillure éliminées par le pré-rinçage (estimées par analyse des différents composants du lait) sont très voisines. Comme le calcul théorique montre que la géométrie des deux installations est très semblable, la plaque verticale est donc souillée comme un tank de stockage.

- Les débits de rinçage de la plaque et des essais standards relatifs au tank de stockage (TISSIER et al., 1983a) sont voisins si le débit est ramené au périmètre souillé. Il est donc possible de comparer directement la vitesse de rinçage des deux dispositifs expérimentaux. Il apparaît que la concentration au début de la deuxième phase de rinçage ne diffère pas significativement pour les deux dispositifs. Cependant, la constante de vitesse de la deuxième phase de la cinétique de rinçage de la plaque est nettement plus élevée que celle observée avec le tank. Ce phénomène est d'autant plus marqué que le composé est éliminé rapidement. Nous avons donc supposé que le film de rinçage formé dans le tank de stockage n'est pas homogène au tout début du rinçage ; chaque point de la surface n'étant pas 
rincé en même temps, la souillure n'est pas éliminée de la surface de façon homogène. Comme la vitesse locale d'élimination de la souillure pendant la deuxième phase est proportionnelle à la quantité de souillure restant à la paroi en chaque point, il en résulte une augmentation de la concentration instantanée de l'effluent après le début du rinçage, et une diminution de la constante de vitesse apparente de rinçage. Il est logique de noter que ce phénomène est donc d'autant plus marqué que le composant de la souillure considéré tend à être éliminé rapidement. La rapidité du rinçage est donc conditionnée par la vitesse d'établissement d'un film tombant de rinçage homogène. Celui-ci se met en place très rapidement dans le cas du rinçage de la plaque.

La plaque expérimentale permet donc de simuler la souillure d'un tank de stockage. La vitesse de rinçage observée dans ces conditions correspond au maximum réalisable lorsque le rinçage est effectué sous l'action d'un simple film tombant. Il faut cependant rappeler que l'enlèvement des composants difficilement éliminables est accéléré si des jets, dirigés sur toute la surface souillée augmente la turbulence du film tombant (Tissier et al., 1983b).

\section{F. Comparaison des cinétiques de rinçage de la plaque et des canalisations}

Notre travail montre que la troisième phase du rinçage des canalisations pendant laquelle les différents composants de la souillure présentent un comportement différent correspond à la deuxième phase du rinçage de la plaque. Les conclusions de Plett et Loncin (1981) qui précisent que les volumes morts, même s'ils sont très petits semblent jouer un rôle important sont en accord avec nos résultats et notre modèle qui prend en compte la rugosité de la surface. De même, après avoir supposé qu'un phénomène de diffusion contrôlait cette phase (THOR et LoNCIN, 1978), PletT et Loncin (1981) ont conclu, comme nous, que la diffusibilité des composants de la souillure n'influence pas de façon marquée cette phase.

Il apparaît également que la quatrième phase du rinçage des canalisations qui correspond à la désorption des composants de la surface selon PLETT (1984), correspond à la dernière phase du rinçage de la plaque. Ainsi, nous observons également que cette phase prend une importance marquée pour les macromolécules chargées électriquement qui peuvent être facilement adsorbés sur la surface.

Il semble donc que les deux dernières phases du rinçage des canalisations et de la plaque soient contrôlées par les mêmes phénomènes. La quantité de souillure initiale de la plaque étant très faible, il est plus facile avec notre dispositif de mettre en évidence ces phases qui concernent de très petites quantités de souillure. Cependant notre dispositif nécessite de prendre en compte la répartition de la souillure sur la plaque, répartition qui suit théoriquement un profil parabolique. De plus, il est impossible de mesurer directement la quantité de souillure restant sur la plaque après rinçage.

\section{Conclusions}

Le dispositif expérimental utilisé qui ne nécessite qu'une faible quantité de souillure permet de simuler la surface d'un tank de stockage. Une étude détaillée de 
la cinétique de rinçage a permis de proposer un modèle qui prend en compte la rugosité de la surface. Ce modèle comprend trois phases consécutives qui sont influencées par la concentration et la nature de la souillure utilisée :

- La première phase, décrite par un modèle réactionnel d'ordre zéro correspond à l'élimination de la souillure dont l'épaisseur sur la plaque est plus grande que la profondeur des rugosités de la surface. Pendant cette phase, une souillure donnée est éliminée à vitesse constante.

- La deuxième phase, décrite par un modèle réactionnel du premier ordre, correspond à l'élimination de la souillure contenue dans les rugosités de la surface. Le film tombant de rinçage ne pénètre pas les cavités présentes sur la plaque dont la concentration en souillure reste homogène. L'élimination de la souillure, qui s'effectue selon la première loi de FICK, entraîne une diminution progressive de la concentration en souillure des cavités. Lorsque l'épaisseur initiale de souillure présente sur la plaque est plus faible que la profondeur des rugosités de la surface, il se produit, lors de l'établissement du film tombant, un remplissage instantané des cavités par l'eau de rinçage qui dilue la souillure contenue dans celle-ci.

- La troisième phase du rinçage, qui se manifeste par un ralentissement de la cinétique, correspond à l'élimination de très faibles quantités de souillure restant sur la plaque. Notre dispositif expérimental s'est révélé mal adapté à une étude précise de cette dernière phase qui peut être décrite empiriquement par une augmentation de l'ordre de la cinétique de rinçage. Ce phénomène pourrait être contrôlé par un mécanisme de désorption des composants macromoléculaires de la souillure (protéines, globules gras) ou lié à la présence de faibles quantités de souillure, de viscosité élevée, " piégée " dans des microrugosités plus profondes de la surface.

Les composés simples ionisés (chlorure de sodium, acide nitrique, soude) et ceux qui ne présentent pas de charges électriques (lactose, saccharose) sont rapidement éliminés pendant le rinçage. La durée de rinçage efficace dépend de la quantité de souillure restant à la paroi et donc de la viscosité de la solution de souillure.

Les protéines et la matière grasse du lait sont rincées plus lentement. L'homogénéisation ralentit la vitesse de rinçage de la matière grasse du lait qui devient voisine de celle des micelles de caséine du lait. Le comportement des protéines du lactosérum et de la matière grasse non homogénéisée paraît intermédiaire.

Reçu le 19 janvier 1987 Accepté pour publication le 18 février 1988

\section{Références bibliographiques}

ATkıns P.W., 1982. Chimie Physique, Technique et Documentation - Lavoisier, Vuibert Eds.

Bird R.B., Stewart W.E., Lightfoot E.N., 1960. Transport Phenomena, John Wiley and Sons Eds.

Kessler H.G., 1981. Food Engineering and Dairy Technology, VerlaG A. Kessler Eds.

Liley P.E., Gambill W.R., 1973. Physical and Chemical Data. In : Perry R.H., Chilton C.H. ed. Chemical Engineers Handbook, 5e Edition, Mc Graw-HILL eds, part 3, 224-225. 
LoEwUS F.A., 1952. Improvement in anthrone method for determination of carbohydrates. Anal. Chem., 24, 219.

Lowry O.H., Rosebrough N.J., Farr A.L., Randal R.J., 1951. Protein measurement with the Folin phenol reagent, J. Biol. Chem., 193, 265-275.

NAssauer J., Kessler H.G., 1982. Problems with cleaning and rinsing of pipelines and fittings. Z, Lebensmittel - Technol. Verfahrenstech., 2, 77-85.

Plett A.E., Loncin M., 1981. The rinsing problem in the food industrie. In : Fundamentals and Applications of Surface Phenomena Associated with Fouling and Cleaning in Food Processing Proceedings - Hallstrom B., LuND D.B., TRAGARDH CH. eds. - Lund University - Alnarp (Sweeden), 365-377.

PLeTt A.E., 1984. Rinsing kinetics of fluid food equipment. In : Engineering and Food, Vol. 2. Processing Applications, McKenna B.M. Ed., Elsevier Applied Science Publishers London and New York, 659-668.

SCHLUSSLER H.J., KoCH F., 1979. Zur Frage der Kontamination von Lebensmitteln durch Reinigungs und Desinfektionsmittel unter praktischen Betriebsbedingungen. Monatsschr. Brau., 32, 80-88.

Schmidt D.G., 1980. Colloidal aspect of casein. Neth. Milk Dairy J., 34, 42-64.

THOR W., LoNCIN M., 1978. Reinigen, Desinfizieren und Nachspülen in der Lebensmittel Industrie. Chem. Ing. Tech., 50, (3), 188-193.

TISSIER J.P., CORRIEU G., 1983. Dosage par turbidité de la concentration en matière grasse d'effluents de pré-rinçage de tanks de stockage de lait entier. Lait, 63, 58-65.

Tissier J.P., Corrieu G., LAlande M., 1983a. Étude du nettoyage de tank de stockage, I. Description d'une méthode de suivi des différentes étapes du rinçage et du nettoyage. Essai de quantification des phénomènes. Lebensm. - Wiss. Technol., 17, 294-299.

Tissier J.P., Corrieu G., Lalande M., 1983b. Étude du nettoyage de tank de stockage. II. Évaluation de quelques paramètres susceptibles d'influencer la cinétique du pré-rinçage. Lebensm. Wiss. Technol., 17, 300-307.

TISSIER J.P., 1984. Étude du rinçage d'un réservoir de stockage. Aptitude au rinçage de produits laitiers, du saccharose et de solutions de nettoyage. Thèse de Docteur-Ingénieur DI 141, Université de Clermont-Ferrand II.

Van-Rossum J.J., 1958. Viscous lifting and drainage of liquids. Appl. Sci. Res. A7, 121-144.

\section{NONEMCLATURE}

\section{Variables :}

C : Concentration $\left(\mathrm{kg} \cdot \mathrm{m}^{-3}\right)$.

$\mathrm{C}_{\mathrm{o}} \quad$ : Concentration de la souillure initiale $\left(\mathrm{kg} \cdot \mathrm{m}^{-3}\right)$.

$\mathrm{C}_{\mathrm{f}} \quad$ : Concentration en souillure du film tombant $\left(\mathrm{kg} \cdot \mathrm{m}^{-3}\right)$.

$\mathrm{C}_{\mathrm{s}} \quad$ : Concentration du film de souillure pendant le rinçage $\left(\mathrm{kg} \cdot \mathrm{m}^{-3}\right)$.

D : Coefficient de diffusivité $\left(\mathrm{m}^{2} \cdot \mathrm{s}^{-1}\right)$.

e : Epaisseur du film tombant de rinçage $(\mathrm{m})$.

$\mathrm{e}_{\mathrm{o}} \quad$ : Epaisseur initiale de souillure $(\mathrm{m})$.

$e_{p} \quad$ : Profondeur des cavités (m).

$\mathrm{e}_{1} \quad$ : Epaisseur de la couche limite équivalente $(\mathrm{m})$.

E.T.M. : Ecart type de la moyenne.

$\mathrm{g} \quad$ : Accélération de la pesanteur $\left(\mathrm{m} \cdot \mathrm{s}^{-2}\right)$.

$\mathrm{h}$ : Hauteur de la plaque $(\mathrm{m})$.

$\mathrm{k} \quad$ : Constante de vitesse d'une réaction du premier ordre $\left(\mathrm{s}^{-1}\right)$.

M : Moyenne.

n : Ordre réactionnel. 
Q : Débit $\left(\mathrm{m}^{3} \cdot \mathrm{h}^{-1}\right)$.

$\operatorname{Re} \quad$ : Nombre de Reynolds.

S : Surface $\left(\mathrm{m}^{2}\right)$.

$\mathrm{t} \quad$ : Durée (s).

$\mathrm{t}_{\mathrm{s}} \quad$ : Temps de séjour moyen du film tombant sur la surface souillée (s).

$\mathrm{v} \quad$ : Vitesse de déplacement $\left(\mathrm{m} . \mathrm{s}^{-1}\right)$.

$\gamma \quad$ : Viscosité cinématique $\left(\mathrm{m}^{2} \cdot \mathrm{s}^{-1}\right)$.

$\Gamma \quad$ : Débit de rinçage par unité de largeur de plaque souillée $\left(\mathrm{m}^{2} . \mathrm{s}^{-1}\right)$.

Indices :

o : Origine.

1 : Première phase du rinçage.

2 : Deuxième phase du rinçage.

3 : Troisième phase du rinçage.

Exposant :

$+\quad$ : Variable réduite (ND). 